\title{
AEEG DEVELOPMENTAL DEVELOPMENTAL CHANGES DURING THE FIRST THREE DAYS AFTER BIRTH IN VERY PRETERM INFANTS
}

\author{
G. Natalucci $^{1,2}$, C.F. Hagmann ${ }^{1}$, V. Bernet ${ }^{3}$, H.U. Bucher ${ }^{1}$, V. Rousson ${ }^{4}$, B. Latal ${ }^{2}$ \\ ${ }^{1}$ Division of Neonatology, University Hospital Zurich, ${ }^{2}$ Child Development Center, ${ }^{3}$ Department of Pediatric \\ Intensive Care and Neonatology; University Children's Hospital Zurich, Zurich, ${ }^{4}$ Institute for Social and \\ Preventive Medicine, University Hospital and Faculty of Medicine, Lausanne, Switzerland
}

Background: Maturation of amplitude-integrated electroencephalogram (aEEG) activity in the preterm infant has been shown to be influenced by both gestational and postmenstrual age.

Objective: To describe the development of aEEG activity in preterm infants $<32$ gestational weeks over the first three days of life in relation to the presence of cerebral injuries.

Methods: 104 infants born at 25-31 gestational weeks without evidence of congenital abnormalities had continuous two-channel aEEG monitoring during the first $72 \mathrm{~h}-84 \mathrm{~h}$ of life. aEEG characteristics were evaluated visually applying pre-established criteria ("background pattern", "maturity score") and quantitatively (maximum and minimum amplitude). Cranial ultrasound was obtained at day 1, 3, 7, and then repeated on a weekly basis until discharge.

Results: A significant increase in the cortical activity was observed during the monitoring period:

a) aEEG pattern significantly changed from an immature to a more mature pattern. This trend occurred more rapidly in newborns without any brain lesion $(n=75)$ on cranial ultrasound compared with newborns with brain lesions. The sleep-wake cycling activity tended to develop particularly slower in newborns with brain lesions;

b) the maximum and minimum aEEG amplitude increased significantly over time in all newborns;

c) the background score correlated well with the maturity score as well as the maturity score with the quantitative measurements.

Conclusions: aEEG activity matures significantly already during the first days of life. In the presence of overt cerebral lesions this process is delayed. The good correlation between the different aEEG assessment tools are of utility in the clinical practice. 